\title{
STOCHASTIC CONTROLS AND FORWARD-BACKWARD SDES *
}

\author{
Jiongmin Yong \\ Laboratory of Mathematics for Nonlinear Sciences \\ and Department of Mathematics \\ Fudan University, Shanghai 200433, China \\ jyong@ms.fudan.edu.cn
}

\section{INTRODUCTION}

Optimal control theory has more than 40 years of history. For deterministic finite dimensional case, the works of Pontryagin et al (maximum principle [14]), Bellman (dynamic programming [1]) and Kalman (linear quadratic regulator problem [3]) have been regarded as three main milestones in the field. For inifite dimensional (deterministic) theory, we refer the readers to the book by $\mathrm{Li}$ and Yong [4].

For stochastic optimal control theory, we would like to mention the works by Kushner, Haussmann, Bismut, Bensoussan, Fleming, Wonham, and so on. For an extensive discussion and summary, see the forthcoming book by Yong and Zhou [18]. The purpose of this paper is to give a brief survey on the works done by the people from Fudan Group related to stochastic controls and forward-backward stochastic differential equations.

\section{MAXIMUM PRINCIPLE}

We consider the following controlled stochastic system:

$$
d x(t)=b(x(t), u(t)) d t+\sigma(x(t), u(t)) d W(t), \quad x(0)=x_{0} .
$$

The cost functional is given by:

$$
J(u(\cdot))=E\left\{\int_{0}^{T} f(x(t), u(t)) d t+h(x(T))\right\} .
$$

*This work is supported in part by the NSFC, under grant 79790130, the National Distinguished Youth Science Foundation of China under grant 19725106, the Chinese State Education Commission Science Foundation under grant 97024607, and the Li Foundation.

The original version of this chapter was revised: The copyright line was incorrect. This has been corrected. The Erratum to this chapter is available at DOI: 10.1007/978-0-387-35359-3_40 
Control $u(t) \in U$ almost surely, with $U$ being some metric space. The optimal control problem is to minimize cost (2.2) subject to (2.1). We note that the diffusion coefficient $\sigma$ depends on the control variable $u$ and the control domain $U$ is not necessarily convex. The main difficulty in deriving the necessary conditions for the optimal controls is that the Itô integral only guarantees the estimate: $\int_{t}^{t+\varepsilon} \sigma d W(s) \sim \sqrt{\varepsilon}$, which is not enough if one use the same argument for deterministic problem to treat the stochastic problem (2.1)-(2.2). Peng proved the following result in 1990 [12].

Theorem 2.1. Let $(\bar{x}, \bar{u})$ be optimal. Then there exists an adapted solutions $(p, q),(P, Q)$ of:

$$
\begin{array}{r}
d p=-H_{x}(\bar{x}, \bar{u}, p, q) d t+q d W(t), \quad p(T)=-h_{x}(\bar{x}(T)), \\
d P=-\left[B^{T} P+P B+\Sigma^{T} P \Sigma+\Sigma^{T} Q+Q^{T} \Sigma\right. \\
\left.+H_{x x}(\bar{x}, \bar{u}, p, q)\right] d t+Q d W(t), \quad P(T)=-h_{x x}(\bar{x}(T)),
\end{array}
$$

such that

$$
\Delta H(t)-\frac{1}{2} \Delta \sigma(t)^{T} P(t) \Delta \sigma(t) \geq 0, \quad \text { a.e. , a.s. , }
$$

where

$$
\begin{array}{r}
H(x, u, p, q)=-f(x, u)+p^{T} b(x, u)+q^{T} \sigma(x, u), \\
B=b_{x}(\bar{x}(t), \bar{u}(t)), \\
\Delta H(t)=H(\bar{x}, \bar{u}(t), p, q)-H(\bar{x}, u, p, q), \\
\Delta \sigma(t)=\sigma(\bar{x}(t), \bar{u}(t))-\sigma(\bar{x}(t), u) .
\end{array}
$$

We call (2.3) and (2.4) the first and second adjoint equations, respectively, and (2.5) the maximum condition.

\section{RELATIONS BETWEEN MP AND DP}

We would like to look at the relation between maximum principle (MP, for short) and dynamic programming (DP, for short). Consider the stochastic optimal control problem on $[s, T]$ with $s \in[0, T)$. We introduce the value function:

$$
V(s, y)=\inf _{u(\cdot)} J(s, y ; u(\cdot)) .
$$

The corresponding HJB equation takes the following form:

$$
-V_{t}+\sup _{u \in U} G\left(x, u,-V_{x},-V_{x x}\right)=0,\left.\quad V\right|_{t=T}=h,
$$

where

$$
G(x, u, p, P)=\frac{1}{2} \operatorname{tr}\left[\sigma \sigma^{T}(x, u) P\right]+p^{T} b(x, u)-f(x, u)
$$


Classical result asserts that if $V(t, x)$ is smooth enough and $(\bar{x}, \bar{u})$ is optimal, then

$$
\begin{array}{r}
V_{t}(t, \bar{x}(t))=G\left(\bar{x}, \bar{u},-V(t, \bar{x}),-V_{x x}(t, \bar{x}),\right. \\
=\max _{u \in U} G\left(\bar{x}, u,-V_{x}(t, \bar{x}),-V_{x x}(t, \bar{x}),\right. \\
V_{x}(t, \bar{x}(t))=-p(t), \\
V_{x x}(t, \bar{x}(t)) \sigma(t, \bar{x}(t))=-q(t) .
\end{array}
$$

Since the value function is not necessarily smooth, the above result is jsut formal. Zhou proved the following in 1991 [19]

Theorem 3.1. Let $(\bar{x}, \bar{u})$ be optimal, $V(t, x)$ be the value function, $(p, q)$ and $(P, Q)$ be adapted solutions of adjoint equations. Then

$$
\begin{gathered}
D_{t+, x}^{1,2,-} V(t, \bar{x}(t)) \subseteq(-\infty, \mathcal{H}(t)] \times\{-p(t)\} \times(-\infty,-P(t)], \\
D_{t+, x}^{1,2,+} V(t, \bar{x}(t)) \supseteq[\mathcal{H}(t), \infty) \times\{-p(t)\} \times[-P(t), \infty),
\end{gathered}
$$

where

$$
\begin{array}{r}
\mathcal{H}(t)=\frac{1}{2} \sigma(\bar{x}, \bar{u})^{T} P \sigma(\bar{x}, \bar{u})+p^{T} b(\bar{x}, \bar{u})-f(\bar{x}, \bar{u}) \\
+\sigma(\bar{x}, \bar{u})^{T}[q-P \sigma(\bar{x}, \bar{u})]
\end{array}
$$

and $D_{t+, x}^{1,2, \pm} V$ are the generalized second order right differentials.

\section{VERIFICATION THEOREM}

Verification theorem gives sufficient conditions for an admissible pair to be optimal. The classical result is as follows: Let $v$ be a solution of HJB equation (3.1). Then

$$
v(s, y) \leq V(s, y)
$$

and $(\bar{x}, \bar{u})$ is optimal if and only if

$$
v_{t}(t, \bar{x})=G\left(\bar{x}, \bar{u},-v_{x}(t, \bar{x}),-v_{x x}(t, \bar{x})\right) .
$$

Again, since the value function might be non-smooth, the above result is merely formal. Zhou, Yong and Li proved the following in 1997 [20].

Theorem 4.1. Let $v$ be a viscosity solution of HJB equation (3.1). Then (4.1) holds and $(\bar{x}, \bar{u})$ is optimal if there exists a triple $(\bar{q}, \bar{p}, \bar{P}) \in D_{t+, x}^{1,2,+} v(t, \bar{x}(t))$ $(t \in[0, T])$ such that

$$
E \int_{0}^{T} \bar{q}(t) d t \leq E \int_{0}^{T} G(\bar{x}, \bar{u},-\bar{p},-\bar{P}) d t .
$$

We point out that the above condition is only sufficient. 


\section{FBSDES}

Let $\left(\Omega, \mathcal{F}, P,\left\{\mathcal{F}_{t}\right\}_{t \geq 0}\right)$ be a probability space and $W(\cdot)$ be a $d$-dimensional standard Brownian motion with $\mathcal{F}_{t}=\sigma(W(s), s \leq t)$. Consider

$$
\begin{array}{r}
d X(t)=b(t, X(t), Y(t), Z(t)) d t+\sigma(t, X(t), Y(t), Z(t)) d W(t), \\
d Y(t)=h(t, X(t), Y(t)) d t+\hat{\sigma}(t, X(t), Y(t), Z(t)) d W(t), \\
X(0)=x, \quad Y(T)=G(X(T)) .
\end{array}
$$

Definition 5.1. An adapted solution of (5.1) is a triple of $\left\{\mathcal{F}_{t}\right\}_{t \geq 0 \text {-adapted }}$ processes $(X, Y, Z)$ satisfying the above in the usual sense of Itô integral.

The key issues here are the following: The Itô integral is forward, the second equation is to be solved backwardly, and the solution is required to be forwardly adapted. Two major motivations of studying the above equations are: stochastic maximum principle, and pricing of European type contingent claims.

Note that FBSDEs are not always solvable. Here is an simple example.

$$
\begin{array}{r}
d X(t)=Y(t) d t+\sigma(X(t), Y(t), Z(t)) d W(t), \\
d Y(t)=-X(t)+Z(t) d W(t), \\
X(0)=x, \quad Y(T)=X(T),
\end{array}
$$

where $\sigma$ is any suitable function. For $T=\frac{\pi}{4}+2 k \pi, k \geq 0$ and $x \neq 0$, the above does not have adapted solutions. In fact if $(X(\cdot), Y(\cdot), Z(\cdot))$ were an adapted solution, then $x(t)=E X(t), y(t)=E Y(t)$ would satisfy

$$
\begin{aligned}
\dot{x}(t)=y(t), & \dot{y}(t)=-x(t), \\
x(0)=x, & y(T)=x(T),
\end{aligned}
$$

which is impossible.

We now introduce the Method of Optimal Control to approach the solvability of (5.1). To this end, we consider

$$
\begin{array}{r}
d X=b(X, Y, Z) d t+\sigma(X, Y, Z) d W(t) \\
d Y(t)=h(X, Y, Z) d t+Z d W(t), \\
X(0)=x, \quad Y(0)=y
\end{array}
$$

We regard $(X, Y)$ as the state and $Z \in \mathcal{Z}[0, T] \triangleq L_{\mathcal{F}}^{2}\left(0, T ; \mathbb{R}^{m \times d}\right)$ as the control. Introduce cost functional:

$$
J(x, y ; Z(\cdot)) \triangleq E f(X(T), Y(T)),
$$

with $f$ being Lipschitz and

$$
\begin{aligned}
& f(x, y) \geq 0, \quad \forall(x, y) \in \mathbb{R}^{n} \times \mathbb{R}^{m}, \\
& f(x, y)=0, \quad \text { if and only if } y=g(x) .
\end{aligned}
$$


Problem (OC). For $(x, y) \in \mathbb{R}^{n} \times \mathbb{R}^{m}$, find a $\bar{Z}(\cdot) \in \mathcal{Z}[0, T]$, such that

$$
V^{0}(x, y) \triangleq \inf _{Z(\cdot) \in \mathcal{Z}[0, T]} J(x, y ; Z(\cdot))=J(x, y ; \bar{Z}(\cdot))
$$

Proposition 5.2. For $x \in \mathbb{R}^{n}$, (5.1) admits an adapted solution ifand only if $\exists y \in \mathbb{R}^{m}$, s.t. $V^{0}(x, y)=0$ and there exists an (optimal) control $Z(\cdot) \in \mathcal{Z}[0, T]$, such that

$$
J(x, y ; Z(\cdot))=V^{0}(x, y)(=0) .
$$

Thus, we need to look at the following two sub-problems:

(i) Find $(x, y)$ such that $V^{0}(x, y)=0$;

(ii) Existence of an optimal control for $(x, y)$ such that $V^{0}(x, y)=0$.

Definition 5.3. For $x \in \mathbb{R}^{n},(5.1)$ is said to be approximately solvable if $\forall \varepsilon>0, \exists\left(X_{\varepsilon}, Y_{\varepsilon}, Z_{\varepsilon}\right)$, such that

$$
\begin{array}{r}
d X_{\varepsilon}=b\left(X_{\varepsilon}, Y_{\varepsilon}, Z_{\varepsilon}\right) d t+\sigma\left(X_{\varepsilon}, Y_{\varepsilon}, Z_{\varepsilon}\right) d W(t), \\
d Y_{\varepsilon}=h\left(X_{\varepsilon}, Y_{\varepsilon}, Z_{\varepsilon}\right) d t+Z_{\varepsilon} d W(t), \\
X_{\varepsilon}(0)=x,
\end{array}
$$

$$
E\left|Y_{\varepsilon}(T)-g\left(X_{\varepsilon}(T)\right)\right|<\varepsilon
$$

We call $\left(X_{\varepsilon}, Y_{\varepsilon}, Z_{\varepsilon}\right)$ an approximate adapted solution of (5.1).

Theorem 5.4. Under some mild conditions, (5.1) is approximately solvable if and only if there exists a $y \in \mathbb{R}^{m}$, such that $V^{0}(x, y)=0$.

Let us consider a special case.

$$
\begin{array}{r}
d X=b(X, Y) d t+\sigma(X, Y) d W(t) \\
d Y=h(X, Y) d t+Z d W(t), \\
X(0)=x, \quad Y(T)=g(X(T))
\end{array}
$$

All the coefficients could also be time dependent. The point is that functions $b, \sigma$ and $h$ are independent of $Z$.

Theorem 5.5. Let $g$ be bounded in $C^{2+\alpha}\left(\mathbb{R}^{n}\right), b, \sigma, h$ be $C^{2}$ with bounded first and second derivatives and

$$
|b(x, 0)|+|\sigma(x, 0)|+|h(x, 0)| \leq L, \quad \forall x \in \mathbb{R}^{n}
$$

Then (5.7) is approximately solvable.

The difficulties that need to be overcome are: (i) $\sigma$ might be degenerate, and (ii) The control domain $\left(\mathbb{R}^{m \times d}\right)$ is not compact. 


\section{EUROPEAN TYPE CONTINGENT CLAIMS}

We now look at problem of European type contingent claims, which is very closely related to the BSDEs and/or FBSDEs. Consider the following:

$$
\begin{array}{r}
d X(t)=b(t, X(t)) d t+\sigma(t, X(t)) d W(t), \quad X(0)=x_{0} \\
d Y(t)=[r(t, X(t)) Y(t)+\langle h(t, X(t)), Z(t)\rangle] d t \\
+\langle Z(t), \sigma(t, X(t)) d W(t)\rangle, \quad Y(0)=y_{0} .
\end{array}
$$

In the above, $X(\cdot)$ is the log-price process, and $Y(\cdot)$ is the wealth process. We do not assume the non-degeneracy of $\sigma$. Thus, the market could be incomplete. We introduce the following assumption:

(H) There exist $L>0, \lambda \geq 0$, such that

$$
\begin{array}{r}
|b(t, x)-b(t, \widehat{x})|+|\sigma(t, x)-\sigma(t, \widehat{x})| \leq L|x-\widehat{x}|, \\
|b(t, x)|+|\sigma(t, x)|+|r(t, x)|+|h(t, x)|+e^{-\lambda|x|}|g(x)| \leq L .
\end{array}
$$

For European call option, $g(x)=\left(e^{x}-q\right)^{+}$for some $q>0$. Thus, one can take $\lambda=1$. Also, we note that assuming $g(x)$ to be bounded will exclude many interesting cases.

Now, we introduce the set of all portfolios:

$$
\begin{gathered}
\mathcal{Z}[0, T] \triangleq\left\{Z:[0, T] \times \Omega \rightarrow \mathbb{R}^{n} \mid Z(\cdot) \text { is }\left\{\mathcal{F}_{t}\right\}_{t \geq 0 \text {-adapted }}\right. \\
\left.|\langle h(\cdot, X(\cdot)), Z(\cdot)\rangle|,\left|\sigma(\cdot, X(\cdot))^{T} Z(\cdot)\right| \in L_{\mathcal{F}}^{2}(0, T ; \mathbb{R})\right\}
\end{gathered}
$$

We will identify $\xi \in L_{\mathcal{F}_{T}}^{2}(\Omega ; \mathbb{R})$ with the European contingent claim whose payoff at $t=T$ is $\xi$.

Definition 6.1. Let $X(\cdot)$ be the strong solution of $(6.1)$. We say that $\xi$ is hedgeable, if $Y(T) \geq \xi$, a.s. , and $\xi$ is replicatable if $Y(T)=\xi$, a.s. , where $Y(\cdot)$ is the solution of $(6.2)$ for some $\left(y_{0}, Z(\cdot)\right) \in \mathbb{R} \times \mathcal{Z}[0, T]$.

Clearly, replicatability implies hedgeability. We can prove the following facts

(i) Any bounded $\xi$ is always hedgeable, regardless of the completeness of the market;

(ii) When market is complete, any $\xi \in L_{\mathcal{F}_{T}}^{2}(\Omega ; \mathbb{R})$ is replicatable;

When market is incomplete,

(iii) Some bounded $\xi$ could be not replicatable;

(iv) Some unbounded $\xi$ could be not hedgable.

Based on the above, we now concentrate on contingent claim of form $g(X(T))$ with $g: \mathbb{R}^{n} \rightarrow \mathbb{R}$. Then replicatability of $g(X(T))$ is equivalent to the solvability of the following BSDE:

$$
\begin{array}{r}
d Y(t)=[r(t, X(t)) Y(t)+\langle h(t, X(t)), Z(t)\rangle] d t \\
+\langle Z(t), \sigma(t, X(t)) d W(t)\rangle, \quad Y(T)=g(X(T)) .
\end{array}
$$

It is a linear BSDE with random coefficients with the following main features: 
(i) $\sigma(t, X(t))$ could be degenerate;

(ii) $Y(T)$ depends only on $X(T)$, with $X(\cdot)$ being the solution of an FSDE whose diffusion is compatible with that of (6.7).

The following method is an extension of the so-called Four Step Scheme due to Ma-Protter-Yong [5] in studying the solvability of FBSDEs.

Suppose $(Y, Z)$ is an adapted solution of $(6.7)$. Let

$$
Y(t)=u(t, X(t)), \quad t \in[0, T], \text { a.s. },
$$

where $u(\cdot, \cdot)$ is undetermined. Applying Itô's formula,

$$
\begin{array}{r}
d Y(t)=d[u(t, X(t))] \\
=\left\{u_{t}(t, X(t))+\frac{1}{2} \operatorname{tr}\left[\sigma(t, X(t)) \sigma(t, X(t))^{T} u_{x x}(t, X(t))\right]\right. \\
\left.+\left\langle b(t, X(t)), u_{x}(t, X(t))\right\rangle\right\} d t+\left\langle\sigma(t, X(t))^{T} u_{x}(t, X(t)), d W(t)\right\rangle
\end{array}
$$

Comparing (6.7) with (6.9), we should have

$$
\begin{array}{r}
\sigma(t, X)^{T} u_{x}(t, X)=\sigma(t, X)^{T} Z \\
u_{t}(t, X)+\frac{1}{2} \operatorname{tr}\left[\sigma(t, X) \sigma(t, X)^{T} u_{x x}(t, X)\right]+\left\langle b(t, X), u_{x}(t, X)\right\rangle \\
=r(t, X) u(t, X)+\langle h(t, X), Z\rangle \\
u(T, X(T))=g(X(T)) .
\end{array}
$$

Thus, it suffices to solve:

$$
u_{t}+\frac{1}{2} \operatorname{tr}\left[\sigma \sigma^{T} u_{x x}\right]+\left\langle b-h, u_{x}\right\rangle-r u=0, \quad u(T, x)=g(x) .
$$

If this problem admits a classical solution $u(t, x)$, then

$$
Y(t)=u(t, X(t)), \quad Z(t)=u_{x}(t, X(t)),
$$

gives a price and a portfolio replicating the contingent claim $g(X(T))$.

Under some smooth conditions, (6.10) admits a classical solution.

Open Questions:

(1) Large Investor

(2) Random Coefficients

\section{References}

[1] R. Bellman, Dynamic Programming, Princeton Univ. Press, N.J., 1957.

[2] N. El Karoui, S. Peng, and M. C. Quenez, Backward stochastic differential equations in finance, Math. Finance, 7 (1997), 1-71.

[3] R. E. Kalman, Contributions to the theory of optimal control, Bol. Soc. Math. Mexicana, 5 (1960), 102-119. 
[4] X. Li and J. Yong, Optimal Control Theory for Infinite Dimensional Systems, Birkhäuser, Boston, 1995.

[5] J. Ma, P. Protter, and J. Yong, Solving forward-backward stochastic differential equations explicitly - a four step scheme, Prob. Th. \& Rel. Fields, 98 (1994), 339-359.

[6] J. Ma and J. Yong, Solvability of forward-backward SDEs and the nodal set of HJB equations, Chin. Ann. Math. Ser. B, 16 (1995), 279-298.

[7] J. Ma and J. Yong, Adapted solution of a degenerate backward SPDE, with applications, Stoch. Proc. \& Appl. 70 (1997), 59-84.

[8] J. Ma and J. Yong, Approximate solvability of forward-backward differential equations, preprint.

[9] J. Ma and J. Yong, Forward-Backward Stochastic Differential Equations and Their Applications, in preparation.

[10] E. Pardoux and S. Peng, Adapted solution of backward stochastic equation, Syst. \& Control Lett., 14 (1990), 55-61.

[11] E. Pardoux and S. Tang, The study of forward-backward stochastic differential equation and its application in quasilinear PDEs, preprint.

[12] S. Peng, A general stochastic maximum principle for optimal control problems, SIAM J. Control \& Optim., 28 (1990), 966-979.

[13] S. Peng and Z. Wu, Fully coupled forward-backward stochastic differential equations with arbitrarily large time duration, preprint.

[14] L. S. Pontryagin, V. G. Boltyanskii, R. V. Gamkrelidze and E. F. Mischenko, Mathematical Theory of Optimal Processes, Wiley, New York, 1962.

[15] J. Yong, Finding adapted solutions of forward-backward stochastic differential equations - method of continuation, Prob. Th. \& Rel Fields, 107 (1997), 537-572.

[16] J. Yong, Linear forward-backward stochastic differential equations, Appl. Math. Optim., to appear.

[17] J. Yong, European type contingent claims in an incomplete market with constrained wealth and portfolio, preprint.

[18] J. Yong and X. Y. Zhou, Stochastic Control: Hamiltonian Systems and HJB Equations, Springer-Verlag, New York, to appear.

[19] X. Y. Zhou, A unified treatment of maximum principle and dynamic programming in stochastic controls, Stoch. \& Stoch. Rep., 36 (1991), 137-161.

[20] X. Y. Zhou, J. Yong, and X. Li, Stochastic verification theorems within the framework of viscosity solutions, SIAM J. Control \& Optim., 35 (1997), 243-253. 